\title{
Regional Committee papers
}

The World Health Organization (WHO) Regional Committee for the Eastern Mediterranean is WHO's decision-making body in the Eastern Mediterranean Region. It meets annually with representation from all Member States of the Region. A main function of the Committee is to formulate policies and strategies that provide Member States with guidance on action that needs to be taken to promote and protect health in the Region. This section, Regional Committee papers, comprises extracts from selected papers (Annual Report of the Regional Director, Progress reports, Technical discussions, Technical papers) being presented at the Sixty-second session of the WHO Regional Committee for the Eastern Mediterranean, 5-8 October 2015 in Kuwait. It serves to inform readers of certain key issues related to the regional health priorities that are under consideration by the Committee at its Sixty-second session.

\section{Achievements, challenges and ongoing work: highlights of the Annual Report}

I am pleased to present my annual report of the work of WHO in the Eastern Mediterranean Region, which covers 2014 and the early part of 2015 . As in the past two years, the report focuses on the strategic priorities endorsed by the WHO Regional Committee for the Eastern Mediterranean when I came into office in 2012 ${ }^{1}$. These are: health systems strengthening towards universal health coverage; maternal and child health; prevention and control of noncommunicable diseases; health security and control of communicable diseases; and emergency preparedness and response. Together with these five strategic priorities, which represent the key challenges facing health development in the Region, strong emphasis has been given to managerial reform, improving administrative processes, strengthening compliance and transparency.

The work of WHO continued to be dominated in the past year by the escalating emergency and humanitarian situations in several countries. The magnitude of crises in the Region is unprecedented. An estimated 58 million people are now affected, including 16 million refugees or internally displaced persons. WHO supported acute humanitarian responses in Iraq, Jordan, Lebanon, Libya, Palestine, Syrian Arab Republic and Yemen, while maintaining its efforts, with partners, to strengthen the resilience of health systems in countries with prolonged complex emergencies. The destruction of health facilities, the lack of access to many areas to maintain adequate supplies for both acute and chronic

\footnotetext{
Five annexes relating to Regional Office structure, staffing, meetings, publications and collaborating centres can be found on the Regional Office web site at http://www.emro.who.int/ about-who/annual-reports/.
}

medical conditions, and the fleeing of health personnel and their families have all taken a heavy toll in the past year on the ability of some countries to maintain services. While some donors have continued in their humanitarian commitment and generous support, a major challenge to our ability to maintain an adequate response, together with our health partners, is the lack of sustained funding. In 2015 this has resulted in the closure of health programmes and activities in Iraq and threatens the closure of health services and health programmes elsewhere too.

Nevertheless, we continued to step up our support as crises unfolded. WHO facilitated delivery of medicines and medical supplies to hospitals and health facilities in Gaza, both during and after the conflict in 2014, and led the health cluster in the joint health sector assessment with partners. In Iraq, the capacity of the country office was scaled up with deployment of additional international staff in all areas of expertise and WHO hubs and/or focal points are now established in 19 provinces. Ten mobile clinics were deployed in northern Iraq and, as of May 2015, 3.5 million people have been provided with direct access to essential medicines and medical equipment and more than 5 million children have been vaccinated against polio. In the Syrian Arab Republic, WHO took an innovative approach towards working with a range of partners to ensure access to areas that have been hard to reach. More than 13.8 million medical treatments were delivered, of which a third were to hard-to-reach areas, and we were able to mobilize more than 17000 health care workers to conduct polio and measles immunization campaigns. In Yemen, following the escalation of conflict, WHO distributed 181 tonnes of medicines and medical supplies and more than 500000 litres of fuel between March and end July 2015, as well as safe water and sanitation kits, to maintain operability 
of health infrastructure and facilities for communities and internally displaced people.

The Regional Committee endorsed the need to strengthen emergency preparedness and response through an allhazard and multisectoral approach. By the end of 2014, 19 countries had received our support in reviewing their existing national plans, with a view to adopting the comprehensive approach, and two countries have now finalized national plans. Working closely with the International Humanitarian City, Dubai, WHO has now finalized an agreement with the United Arab Emirates to establish a dedicated WHO humanitarian operations/logistics hub, which will support the rapid procurement and provision of critical medical supplies and equipment to countries experiencing emergencies, both inside and beyond the Region. The regional emergency solidarity fund was established. It will be funded at US\$ 4.9 million for the biennium 2016-2017 which is 1\% of the WHO country budget and will be open to other voluntary contributions. Capacity-building of emergency focal points was supported, and will continue each year as we continue to build up the regional roster of trained experts able to be deployed quickly in an emergency.

Following the discussions and resolutions in the World Health Assembly in May 2015 in the wake of the outbreak of Ebola virus disease in 2014, and in relation to reform and strengthening of WHO's emergency preparedness and response capacities, we have undertaken a close review of our capacities in these areas. As a result we have now undertaken further reform by restructuring and reinforcing technical and managerial capacity in this area in order to ensure appropriate readiness and response at both country and regional levels, as well as effective coordination. Thanks to support from the Government of Jordan, we have established a regional centre for emergencies and polio eradication in Amman. A unit focusing on organizational readiness is being established in the centre in Amman to build capacity and ensure that WHO is ready to respond to public health emergencies in the Region. A second entity based in Cairo and comprising two units, emergency response and coordination, will provide leadership and coordination and ensure an effective response mechanism and timely support to countries in crisis.

The Region has made significant reductions in maternal and child mortality since 1990, as shown by the latest monitoring data for the Millennium Development Goals. However, the levels of reduction fall short of meeting the targets of MDGs 4 and 5 by end 2015. At regional level, the under 5 mortality rate fell by $46 \%$ between 1990 and 2013 (below the global reduction of 49\%), with an average annual reduction of $2.6 \%$. However, this is below the $67 \%$ reduction required to achieve the MDG 4 target by 2015. The maternal mortality rate declined by 50\% between 1990 and 2013 (above the global reduction of 45\%), with an average annual reduction of $3 \%$. This too is below the $75 \%$ reduction required to achieve the MDG 5 target by 2015. The nine countries with the highest burden of maternal and child mortality pushed ahead with implementation of their acceleration plans to reduce this burden further. Nevertheless, most of these countries are unlikely to reach the targets by end of 2015. Although weak health systems, emergencies and shortage of funding are major constraints which will need to be adequately addressed, greater attention and a higher level of political commitment and support needs to be given to reducing child and maternal mortality by these countries, and more effective support is needed from international and regional stakeholders. While WHO will continue to support the implementation of cost-effective, high-impact measures during the next biennium, more attention will be given to supporting Member States in addressing health system challenges and implementing community-based interventions.

The situation with regard to malnutrition in the Region has improved somewhat but the progress is insufficient and more work is needed to ensure that all mothers and children in all countries are adequately nourished to maintain health and development. On average, according to World health statistics 2014, the Region has seen a decrease in the prevalence of undernourishment from $22.6 \%$ in 1991 to $13.6 \%$ in 2012. Since 1990, 13 countries of the Region, more than half, have achieved the MDG 1 target of halving the proportion of people who suffer from hunger. However, only two of these countries (Kuwait and Oman) have also met the target set at the World Food Summit in 1996, although Tunisia is very close to meeting this also. Anaemia rates, especially among women of reproductive age and children, are still high in the Region as are the rates for stunting and for low birth weight. Equally crucial for children, and for long-term health, the proportion of women practising exclusive breastfeeding for at least 6 months continues to be very low. WHO, in coordination with United Nations partners, is supporting Member States on how best to implement the WHO global targets in nutrition and the recommendations of the Second International Conference on Nutrition (ICN-2) held in 2014. A regional framework for action is in the process of development and will comprise a set of policy options and cost-effective interventions to scale up nutrition in the Region.

With regard to MDG 6, while there has been substantial progress in the Region in control and prevention of HIV, malaria and tuberculosis, this has not been enough to achieve the targets. The challenges to health system capacity that the Region faces in general, in particular in countries where the burden of communicable diseases is the highest, as well as the impact of the complex emergencies in the Region, are the main factors affecting further progress. 
The overall prevalence of HIV remains low in comparison with other regions, but it is important to recognize that the number of new HIV infections continues to increase in key population groups who are at increased risk of HIV. Receiving antiretroviral therapy, which is crucial to maintaining quality of life and preventing new infections, remains far below global targets. We continued to support countries in 2014 to implement the regional initiative to end the HIV treatment crisis, and Member States should continue to place emphasis on this.

The regional burden of malaria has decreased substantially, with a halving of the number of deaths since 2000, along with the number of affected countries. Seven countries have achieved the malaria-related target of MDG 6, while in five countries, a reliable assessment of trends is not feasible owing to inconsistent reporting of malaria information. A regional action plan, to implement the global strategy for malaria 2016-2030 has been developed, with the aim of interrupting malaria transmission where feasible and reducing the burden by more than $90 \%$ where elimination is not immediately feasible.

While the Region has achieved the MDG targets of halting and reversing the tuberculosis incidence, it has not yet reached the STOP TB targets of halving the prevalence and the mortality. Five countries are contributing to $84.5 \%$ of the regional burden of tuberculosis. Crucially, it is estimated that $40 \%$ of cases are still missed or not reported and this has serious implications for overall control of the disease. In 2014, WHO developed guidance on control of tuberculosis in complex emergencies, as well as a package of services for cross-border patients. We hope that highburden countries will move forward in implementing these important measures in 2015.

The situation with regard to polio eradication continued to be of concern in 2014, with the Region remaining endemic and accounting for $99 \%$ of all cases reported globally in the second half of the year. However, in 2014 the groundwork was laid for progress in polio eradication in the Region in 2015. By the end of 2014, Pakistan and Afghanistan had developed and were implementing accelerated plans for the low transmission season. The response of the Region to the outbreak in the Middle East in 2013 was swift and of high quality with 25 million children immunized, in multiple campaigns in eight countries. The outbreak was contained in 36 weeks, despite the complex emergency situation in the Region, with the last case reported in April 2014 and so more than one year has passed with no further confirmed cases. Meanwhile the response to the outbreak in the Horn of Africa had, by the end of 2014, reduced transmission to a small pocket.

The national programmes in Afghanistan and Pakistan maintained their commitment to eradication, and health workers and volunteers continued to demonstrate great courage in carrying out immunization activities in difficult situations. As of 19 August 2015, Pakistan and Afghanistan together have reported 36 cases due to wild poliovirus, versus 123 confirmed cases as at the same date in 2014, an overall reduction of nearly $70 \%$. With wild poliovirus now restricted to just Pakistan and Afghanistan, progress will remain fragile until all children in these last foci of endemic circulation are reached and immunized. Full implementation of the acceleration plans remains critical to making progress with eradication in 2015. The countries of the Region are committed to the global plan for the polio-endgame, with all those currently using only oral poliomyelitis vaccine (OPV) on track for introduction of IPV in 2015.

Health security continued to be high on the agenda throughout the past year, and its importance was brought home to governments and the public yet again as the outbreaks of Ebola virus disease spread in three countries in west Africa. The possibility of importation to the Region was a major concern to us. In response to a recommendation from the Regional Committee, WHO urgently undertook a comprehensive assessment of Member States' capacity to deal with a potential importation of Ebola. Between November 2014 and February 2015 rapid assessments of preparedness and readiness measures were conducted by WHO technical teams in 20 countries.

The assessments identified critical weaknesses in the areas of prevention, early detection and response, and a 90-day action plan was subsequently implemented in the Region, starting in May 2015, to assist countries to bridge the urgent gaps. Many of the gaps identified during the assessment concern the overall ability of countries to implement the core capacities required under the International Health Regulations (IHR 2005). The final deadline for ensuring that national core capacities are in place to implement the Regulations is June 2016. Major weaknesses remain. All countries should reinforce their efforts, in light of the assessments, to address the gaps. WHO's strategic focus for country support includes emphasis on multisectoral coordination which will be crucial to addressing these gaps. The fourth annual meeting of IHR stakeholders, which will take place in late 2015, will review the implementation of national and regional plans for strengthening IHR implementation in the context of Ebola.

While Ebola was a vivid and real health threat from outside the Region, there are other more immediate health threats within the Region. The Middle East respiratory syndrome coronavirus MERS-CoV and the avian influenza $\mathrm{H} 5 \mathrm{~N} 1$ virus represent emerging health threats for which countries need to be prepared as both viruses have pandemic potential. An increase of MERS-CoV cases in two countries in the Region in 2014 owing, primarily, to secondary and nosocomial transmissions in health care 
settings, highlighted the need to ensure the safety of patients and health workers, and to improve infection prevention and control in hospitals and other health facilities and the need to build capacity to care for patients with high-risk infections.

In view of these two emerging health threats, $\mathrm{WHO}$ conducted technical missions - in several countries throughout 2014 and during the earlier part of 2015 to assess the risks and support containment of the outbreaks in hospital settings. A number of capacity-building activities was also conducted which resulted in finalization and rapid implementation of preparedness plans for enhancing surveillance for rapid detection and improving infection prevention and control practices for MERS-CoV and other novel respiratory diseases across all health care settings in the Region. Risk communication plans were developed and rapidly scaled up to raise public awareness among pilgrims, health care workers and the general public and prevent international spread, particularly during the hajj. We continue to seek to fill the gaps in knowledge about MERS-CoV so that public health understanding of the epidemiology and transmissibility of the virus and the effectiveness of the global health response can be improved. In May 2015, we held the fourth in a series of international scientific meetings on this subject since 2013. These have helped the international scientific community to pinpoint the gaps we face in knowledge and information about the mode and risk factors for transmission of this emerging viral infection in humans, as well as to identify the most essential public health measures to effectively halt the transmission and spread of the virus.

Antimicrobial resistance is a rapidly increasing risk for global health security which the Member States of the Region are only just beginning to recognize and acknowledge. The problem has serious implications in the Region and requires urgent action. In continuation of the work we began on antimicrobial resistance in 2013, a rapid country assessment of the situation in the Region was conducted in 2014 to which only 12 countries contributed. The results showed significant gaps in the systems and actions needed at country level to address the threat. The subsequent global report highlights the lack of information on the situation in countries of the RegionWhile the work on producing a detailed country situation analysis covering human and animal health continue, we also started work on an operational framework to support countries in developing action plans for discussion in a high-level multisectoral ministerial meeting that we plan to organize early in 2016.

Member States, through the Regional Committee, have acknowledged the serious magnitude of cardiovascular disease, cancers, diabetes and chronic lung disease and have approved a regional framework of action based on the
United Nations Political Declaration of 2011. However, despite the urgent need to launch strong and comprehensive action, implementation of the key commitments in the regional framework for action remains generally inadequate and is not commensurate with the seriousness of the problem in the Region. WHO is working with Member States on several important initiatives to implement the key commitments included in the four areas of the framework: governance, surveillance, prevention, and health care.

WHO's work in 2014 resulted in the development of technical guidance for implementing the most cost-effective measures or "best buys" in prevention and technical support was provided to many countries. Policy statements and guidelines on reducing salt and fat intake have been developed and are now guiding countries in taking appropriate action based on best practice. A monitoring scheme has been launched to track the progress countries of the Region are making. International experience in integrating common conditions into primary health care was reviewed in an intercountry meeting. This work will continue in 2015 with special emphasis placed on developing technical guidance based on evidence and best practice in the area of health care. We are also working with international experts, including Georgetown University, to support updating of fiscal and legal interventions to help control risk factors and promote better care.

Following endorsement in 2012 and 2013 by the Regional Committee of strategies and actions for health systems strengthening, countries were urged in 2014 to implement the framework for action for progressing towards universal health coverage. Several countries have taken important steps in this regard and all countries now have a clearer picture of what is needed to address the challenges. This knowledge was boosted following the development of pharmaceutical sector country profiles, which highlighted gaps in key areas related to regulations for medicines, access to medicines, selection, procurement, dispensing and rational use.

An in depth survey of medical education is providing valuable insight also on the way forward for countries to improve planning for future needs in the health sector. One area that poses a specific challenge is the acute shortage of, and the need to scale up production of, family physicians in most countries of the Region. Efforts are currently under way to identify evidence-based short- and long-term interventions to overcome the shortage of this group of health care providers. WHO has conducted a comprehensive review of the status of nursing and midwifery in the Region in 2015, to provide clear strategic directions for strengthening this area of work. A framework comprising a list of priority actions will be recommended, taking into account the range of challenges encountered by the different groups of 
countries. Reinforcing nursing and midwifery will continue to be a priority for $\mathrm{WHO}$ in 2015 and over the coming biennium.

Two key achievements were made in the area of health information, which is so critical to health planning and policy development and implementation. The Regional Committee took an important step forward in 2014 with the endorsement of the framework for health information systems and the core indicators. This product was the result of intensive work over the past 2 years with different sectors in Member States and international experts. All countries need to strengthen their health information systems and the challenge for all of them is to implement the framework and to address the gaps in generating and using data for the 68 core indicators. Assisting countries in this task will be the main task for WHO during the next biennium.

The second achievement was the work done so far in strengthening civil registration and vital statistics systems, with special focus on improving the reporting of causespecific mortality. Rapid assessments were completed in 21 countries and comprehensive assessments in 17 countries. Our region currently has the most comprehensive information on the status of CRVS in Member States. Countries have been informed of the existing gaps and urged to address them based on the regional strategy, endorsed by the 60th session of the Regional Committee. While we expand the regional capacity in collaboration with other UN agencies, further focus is required within the health systems on the generation of valid mortality and cause of death data.

2014 has also been an important period for WHO management reform as we continued to implement our commitment to greater effectiveness, accountability and transparency. The timely preparation of the programme budget for 2016-2017, and the early involvement of partners through the bottom-up approach, resulted in improved joint planning, a more focused number of identified priorities and a budget allocation in line with countries' priorities. An improvement in priority-setting and planning skills has been evident.

We continued to support the strengthening of Member State's participation in the governance of $\mathrm{WHO}$, providing high-level briefings for representatives of Member States and permanent missions in Geneva prior to each major meeting of WHO's global governing bodies - the World Health Assembly and the WHO Executive Board. These briefings have proved their value in the contribution of Member States of the Region in the global discussions on health and the work of the governing bodies.

Efforts to strengthen WHO country presence also continued, with emphasis on improving technical expertise and overall management. Country office capacities were assessed in relation to the six categories of work to ensure the presence of strategic and technical leadership capabilities. 2014 saw a significant expansion in technical capacity in several country offices, while in 2015 we have focused on enhancing general management and administrative capacity in the field. A specific strategy was developed to increase compliance in a number of areas of our work, across all our offices, including performance management and adherence to staff rules and regulations. Improving compliance will remain a top priority over the coming years.

The complexity of the operational and security issues in the Region continues to create challenges and constraints for WHO operations, both technically and managerially. The situation in the Region generally has also had an adverse impact on our ability to attract new experienced staff to handle the growing workload and response needs in all areas of our work with Member States. While we are taking steps to address this, we also took steps to support the future needs of countries in several key areas.

Strengthening public health capacity in Member States continued to receive priority. Following the launch in 2013 of a regional initiative to assess public health capacity in countries, two country assessments were successfully conducted, with the support of WHO and a team of international public health experts. The assessment tool was reviewed in early 2015 and will be further refined prior to expanding the initiative further. An increasing number of Member States are asking WHO to conduct the assessment and assist them in implementing its recommendations.

A leadership for health programme was also launched, in early 2015, with the aim of developing future public health leaders who can address, in a proactive way, national and local health problems that have direct impact on population health, and play active roles in the global public health sphere. The first four-week course, conducted in two parts in two locations (Geneva and Muscat), was a great success and highly rated by the participants. The second course will commence towards the end of 2015.

We have also continued to host the annual regional seminar on health diplomacy. This has proved highly successful in bringing together representatives of health and foreign affairs, parliamentarians and academia in discussions around the intersection between health and other sectors. Successive seminars have shown the continuing importance of this kind of dialogue for raising awareness and understanding of the key health issues facing our world, and the role that all sectors have in health diplomacy, globally, regionally and nationally. We will continue to support countries in their efforts to build this capacity and in their efforts to improve the health of the people in the Region. 